Annals of International Medical and Dental Research

E-ISSN: 2395-2822 | P-ISSN: 2395-2814

Vol-8, Issue-1 | January-February 2022

DOI: 10.53339/aimdr.2022.8.1.41

Page no- 325-332 | Section- Research Article (Paediatric Neurosciences)

\title{
Short Term Outcome in Sleep Disturbance Intervention in Children with ASD in a Tertiary Care Hospital in Bangladesh
}

\author{
Shaoli Sarker ${ }^{*}$, Mustafa Mahbub $^{2}$
}

\begin{abstract}
${ }^{1}$ Assistant Professor, Department of Paediatric Neurosciences, Bangladesh Institute of Child Health, Dhaka Shishu (Children) Hospital, Dhaka, Bangladesh. Email: sarkershaoli@gmail.com Orcid ID: 0000-0002-1150-7187

2Professor, Department of Paediatric Neurosciences, Bangladesh Institute of Child Health, Dhaka Shishu (Children) Hospital, Dhaka, Bangladesh.

Email: mm231956@gmail.com

Orcid ID: 0000-0003-1247-293X

*Corresponding author
\end{abstract}

Received: 27 August 2021

Revised: 12 November 2021

Accepted: 21 November 2021

Published: 22 December 2021

\section{Abstract}

Background: Sleep issues are common in people specially in children. The major sleep issues are widespread in children with autism spectrum disorder (ASD) and that poor sleep exacerbates troublesome behavior, such linkages have gotten little attention in both research and therapeutic practice.

Aim: The aim of this study was to identify the relationship between sleep patterns and behavioral difficulties in children with ASD using sleep disturbance treatments. Methods: We selected a total 41 children with sleep disorders as study population. The sleep services (behavioral sleep management techniques) were given by the child development center team of Dhaka Shishu Hospital comprising of physician, developmental therapist and psychologist. Children were split into eight groups and two gender divisions based on their age, and they were then studied over a period of 6 months . Sleep disorders were investigated both before and after intervention. Results: The results revealed that the children's sleep dysfunction improved from before, with the lowest improvement percentage decreasing from 62.9 percent to 51.8 percent and the greatest improvement percentage increasing from 100 percent to 59.2 percent, whereas a sleep problem showed no change at all (sleepwalking). Conclusion: The study has tried and succeeded to an extent to intervene in the sleep dysfunction process of children with ASD in a tertiary care hospital. However, there is still much to learn about the clinical efficacy of these types of behavioral interventions in children with ASD who have sleep disruption.

Keywords:- Sleep, Children, ASD, Study, Percent, Disruption, Sleep issues.

\section{INTRODUCTION}

Around 1 in every 160 children affected, autism spectrum disorder (ASD) is a developmental disease marked by social communication difficulties as well as repetitive and stereotyped interests and activities. [1] Interestingly, children who have a history of developmental regression have a more disrupted sleep pattern than children who do not have a history of regression. Children and adolescents with ASD have a higher proportion of sleep issues, notably insomnia, than typically developing children, ranging from $40 \%$ to $80 \%$.2] Even though sleep regulation in children with ASD is still poorly understood, circadian anomalies in autism may be the result of genetic abnormalities related to melatonin synthesis and melatonin's involvement in modulating synaptic transmission. Recently, a bifurcation of the sleep/wake cycle has been proposed, with 
Annals of International Medical and Dental Research E-ISSN: 2395-2822 | P-ISSN: 2395-2814 Vol-8, Issue-1 | January-February 2022 DOI: 10.53339/aimdr.2022.8.1.41

Page no- 325-332 | Section- Research Article (Paediatric Neurosciences)

heightened sensitivity to external noise and short sleep duration creating inconsistent sleep start and wake up times.[3] Identifying and treating sleep issues may result in not just better sleep, but also better daytime behavior and family functioning. Several studies have also shown that behavioral therapies for sleep onset and maintenance issues in these people are helpful. [4] Disrupted sleep is currently one of the most common complaints among parents of autistic children, with more than 4080 percent of children suffering from sleep problems, compared to $25-40$ percent in typically developing children.[5] Sleep performs several purposes in a developing child, including energy conservation, brain growth, memory consolidation, and cognition. Given the importance of sleep-in daily functioning, interrupted sleep-in people with ASD can have major consequences. Inadequate sleep, according to recent researches, exacerbates the severity of core ASD symptoms (e.g., repetitive behaviors, social and communication issues), as well as other maladaptive behaviors (e.g., self-injury, tantrums, and aggression).[6] To yet, however, there is little evidence of a link between sleep patterns and behavioral issues in people with ASD.

\section{Objective}

The objective of this study is to find a link between sleep patterns and behavioral issues through sleep disturbance intervention in children with ASD.

\section{MATERIAL AND METHODS}

Data were collected from parents of children with ASD who presented at Child Development Center of Dhaka Shishu Hospital with sleep related complaints.The sleep services were provided by a multidisciplinary team comprising of child health physician, developmental therapist and psychologist. Ethical issues were addressed duely. The number of participants was 41, over two years, from 2017 January to 2019 December. Initially, we were willing to take 50 ASD participants with sleep disorders and found more children than we thought for our study. But after observing all of them, we found that although all the children who applied for the study had ASD, not every one of them had a sleep disorder. For this reason, we had to observe them more and cherry-pick only the ones with sleep disturbance- who didn't get any sort of intervention in the past. All data were gathered through parental reporting. The sleep habits of children were thoroughly examined and the parents were given basic training on behavioral sleep management techniques for better results. No sleep medication was prescribed. All the sleep cycle of the children has been recorded through sleep diaries- both noted by the parents and the multidisciplinary team over 6 months period. The variety of sleep profiles in ASD is consistent with ASD samples' heterogeneous phenotypic profiles. Prolonged sleep latency, lower sleep efficiency, increased awakening after sleep onset, daytime tiredness, sleepwalking and aberrant waking time are some of the most frequent sleep difficulties in children with ASD.[7] As a result, there does not appear to be a single sleep disorder that distinguishes children with ASD, but rather a number of them. These sleep challenges appear to remain throughout the lifespan, and individuals with ASD who have one sleep problem frequently have other sleep problems as well.[8] Several of these sleep 
Annals of International Medical and Dental Research

E-ISSN: 2395-2822 | P-ISSN: 2395-2814

Vol-8, Issue-1 | January-February 2022

DOI: 10.53339/aimdr.2022.8.1.41

Page no- 325-332 | Section- Research Article (Paediatric Neurosciences)

problems are recognized as primary sleep disorders by the International Classification of Sleep Disorders (ICSD-3) (e.g., insomnia, parasomnia, and circadian rhythm sleep-wake disorders).[9] For these reasons, we have taken 6 of the most talked-about sleep dysfunctions for our study.

\section{RESULTS}

Data were analyzed for 41 children, aged between 2 to 18 years. $(\mathrm{M}=8.854 ; \mathrm{SD}=3.56)$; whose parents contacted the service, regarding the sleep problem.

While analyzing the data at hand, we can easily come to notice that the number of male children with ASD is greater than that of female ones. In almost all of the cases, we can see that male child are more susceptible to
ASD, but there are some exceptions too. From our study, we can see that the percentage of female children with ASD is greater than the male children in the age range between 8-12 years and 16-18 years. Or at least in our study whatsoever. All the sleep issues have been recorded as per the distribution interval of 2 years. [Table 1]

As we can see from [Table 2], we have identified some of the major sleep issues observed by the parents and the multidisciplinary team. If we look closely into the data, we will be able to see the percentage deviation from the data of the male children and the female children. But the sleep issues remain in such a spot that we cannot at all neglect them.

Table 1: Demographic characteristics of the study people $(\mathrm{N}=41)$

\begin{tabular}{|l|l|l|l|l|l|l|}
\hline \multirow{2}{*}{ Ages (in years) } & Male & Female & \multicolumn{2}{l|}{ Total } \\
\cline { 2 - 7 } & $\mathbf{n}$ & $\mathbf{0}$ & $\mathbf{n}$ & $\mathbf{0}$ & $\mathbf{n}$ & $\mathbf{0}$ \\
\hline $2-4$ yrs. & 2 & 7.41 & 1 & 7.14 & 3 & 7.32 \\
\hline $4-6$ yrs. & 6 & 22.22 & 2 & 14.29 & 8 & 19.51 \\
\hline $6-8$ yrs. & 4 & 14.81 & 2 & 14.29 & 6 & 14.63 \\
\hline $8-10$ yrs. & 4 & 14.81 & 3 & 21.43 & 7 & 17.07 \\
\hline $10-12$ yrs. & 7 & 25.93 & 4 & 28.6 & 11 & 26.83 \\
\hline $12-14$ yrs. & 2 & 7.41 & 0 & 0.0 & 2 & 4.88 \\
\hline $14-16$ yrs. & 2 & 7.41 & 1 & 7.14 & 3 & 7.32 \\
\hline $16-18$ yrs. & 0 & 0.0 & 1 & 7.14 & 1 & 2.44 \\
\hline Total & 27 & 65.85 & 14 & 34.15 & 41 & 100.0 \\
\hline
\end{tabular}

Table 2: Sleep dysfunctions before intervention (behavioral sleep management techniques) $(\mathrm{N}=41)$

\begin{tabular}{|l|l|l|l|l|}
\hline Sleep Issues & \multicolumn{2}{l|}{ Male $\mathbf{( n = 2 7 )}$} & \multicolumn{2}{l|}{ Female $(\mathbf{n}=\mathbf{1 4})$} \\
\cline { 2 - 5 } & $\mathbf{n}$ & $\mathbf{0}$ & $\mathbf{n}$ & $\mathbf{0}$ \\
\hline Prolonged sleep latency & 17 & 62.96 & 7 & 50.0 \\
\hline Lower sleep efficiency & 25 & 92.59 & 11 & 78.57 \\
\hline Increased awakening after sleep onset & 27 & 100.0 & 13 & 92.86 \\
\hline
\end{tabular}


Annals of International Medical and Dental Research

E-ISSN: 2395-2822 | P-ISSN: 2395-2814

Vol-8, Issue-1 | January-February 2022

DOI: 10.53339/aimdr.2022.8.1.41

Page no- 325-332 | Section- Research Article (Paediatric Neurosciences)

\begin{tabular}{|l|l|l|l|l|}
\hline Daytime tiredness & 18 & 66.67 & 9 & 64.28 \\
\hline Sleepwalking & 2 & 7.41 & 0 & 0.0 \\
\hline Aberrant waking time & 26 & 96.29 & 14 & 100.0 \\
\hline
\end{tabular}

Distribution of Sleep dysfunctions before intervention

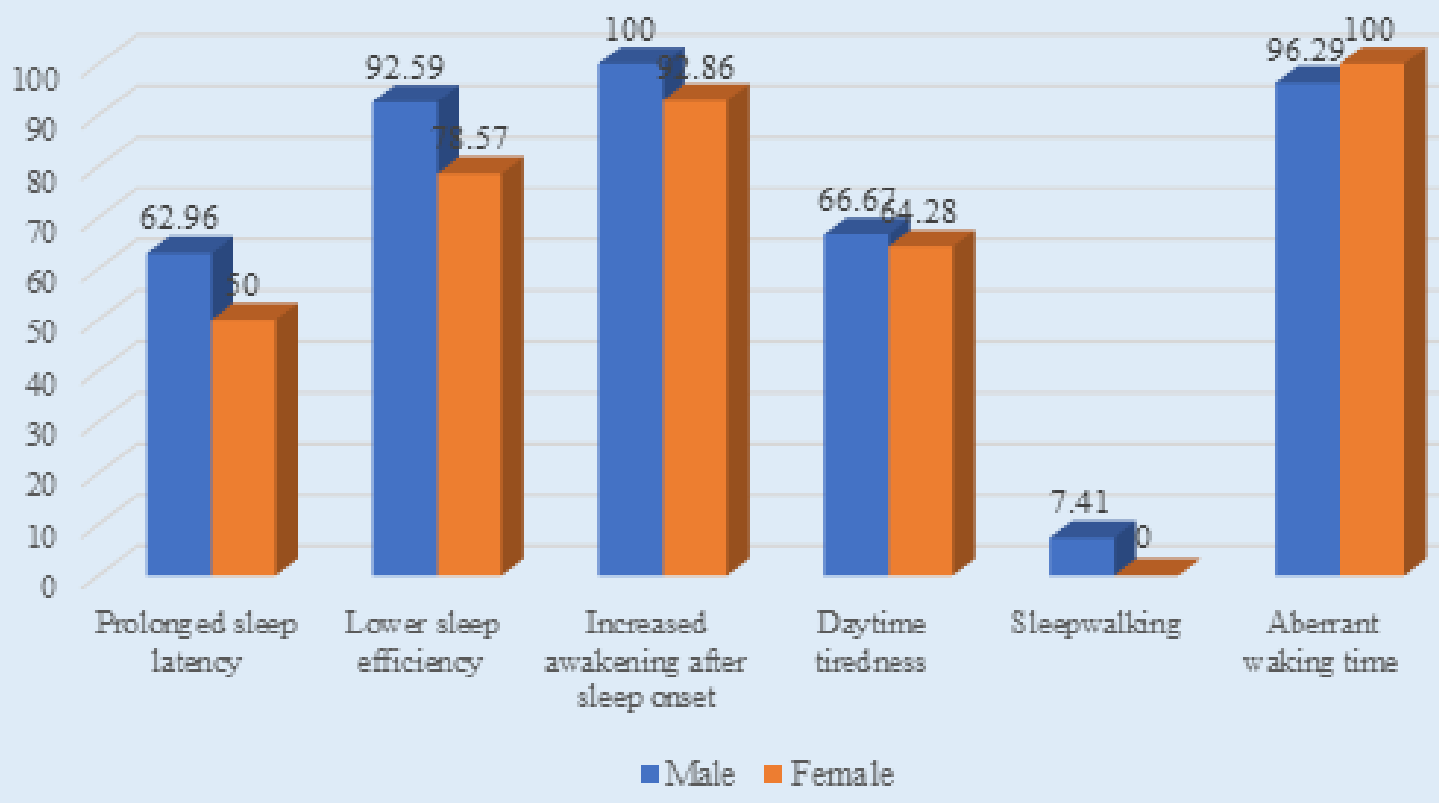

Figure 1: Sleep dysfunctions before intervention 


\section{Distribution of Sleep dysfunctions after intervention}

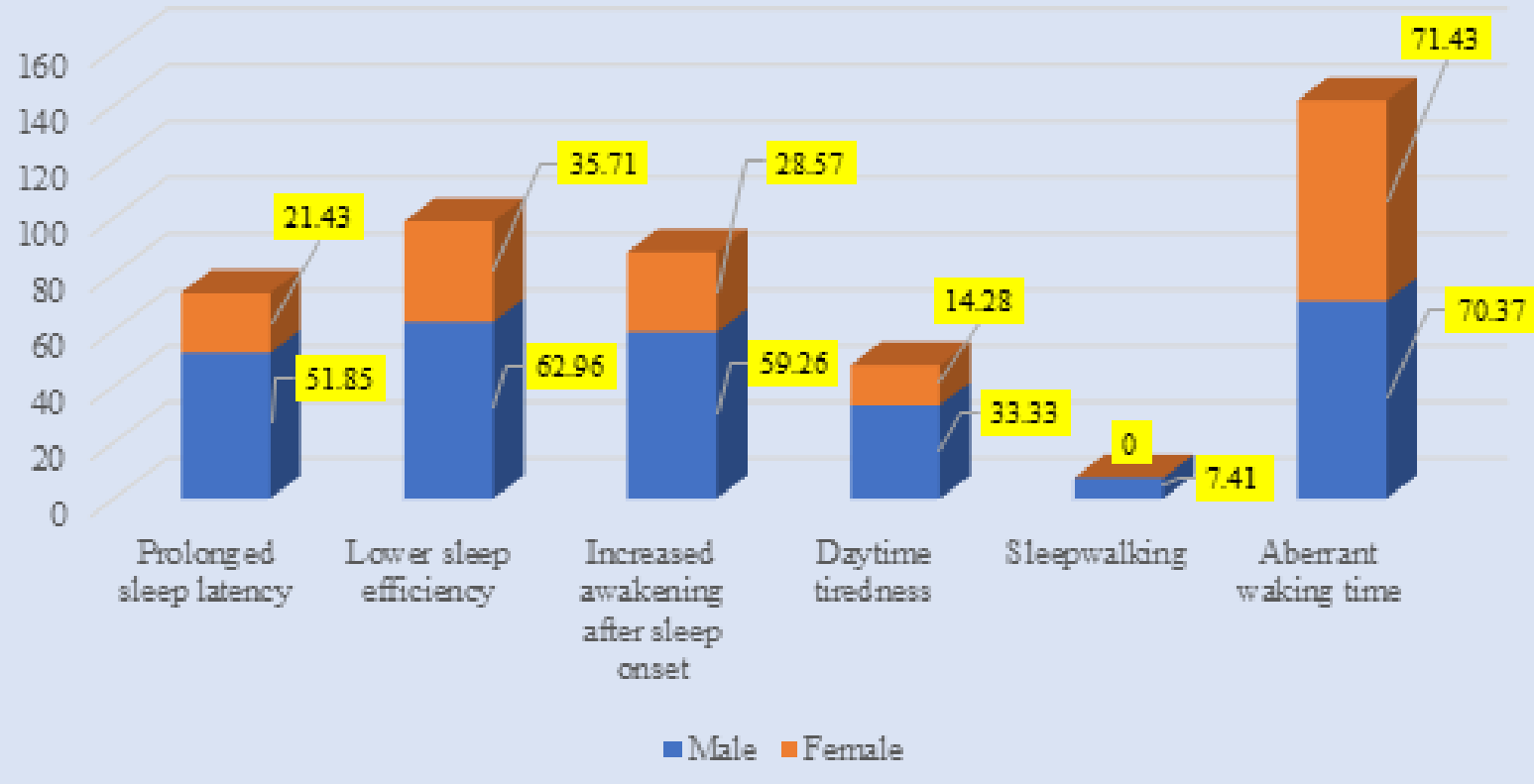

Figure 2: Sleep dysfunctions after intervention

Table 3: Sleep dysfunctions 6 months after intervention

\begin{tabular}{|l|l|l|l|l|}
\hline \multirow{2}{*}{ Sleep Issues } & Male & Female \\
\cline { 2 - 5 } & $\mathbf{n}$ & $\mathbf{0}$ & $\mathbf{n}$ & $\mathbf{\%}$ \\
\hline Prolonged sleep latency & 14 & 51.85 & 3 & 21.43 \\
\hline Lower sleep efficiency & 17 & 62.96 & 5 & 35.71 \\
\hline Increased awakening after sleep onset & 16 & 59.26 & 4 & 28.57 \\
\hline Daytime tiredness & 9 & 33.33 & 2 & 14.28 \\
\hline Sleepwalking & 2 & 7.41 & 0 & 0.0 \\
\hline Aberrant waking time & 19 & 70.37 & 10 & 71.43 \\
\hline
\end{tabular}

As we can see from table 2 and 3 , the percentage of male children having lower sleep efficiency decreased from $92.6 \%$ to $63 \%$. Increased awakening after sleep onset percentage has greatly reduced too, from $100 \%$ to $59.2 \%$ - which is a substantial decrease in percentage. In case of female children, the percentage change is more drastic in case of lower sleep efficiency. So, this behavioral intervention sees a limelight in addressing sleep issues. Although in most of the children, we could not at all demolish the sleep dysfunction issue, we could lessen the issues to one in each child- who had more than one dysfunction. Some of the dysfunctions likeSleepwalking couldn't be lessened at all 
Annals of International Medical and Dental Research

E-ISSN: 2395-2822 | P-ISSN: 2395-2814

Vol-8, Issue-1 | January-February 2022

DOI: 10.53339/aimdr.2022.8.1.41

Page no- 325-332 | Section- Research Article (Paediatric Neurosciences)

through any of our tactics, as we can see from the table. The percentage remains the same before and after. For this reason, we can say that not all dysfunctions can be remedied, or treated better through interventions. This was only a fraction of a study over the entire population of children with ASD, and we have only taken 6 of the sleep issues as our data types. There are several other sleep disorders, that need to be addressed and need further research to understand if they can be eradicated at all or not. [Table 3]

\section{DISCUSSION}

Sleep is a fundamental mechanism for adaptive functioning (e.g., learning, memory, neuroplasticity), it is very likely that sleep impairments play a significant role and it performs better in the case of parentimplemented behavioral sleep treatments for young children with autism spectrum disorder, in the symptoms found in ASD, particularly the aggravation of difficult behaviors.11 Clinically, behavioral sleep treatments are recognized as the first line of treatment for children with ASD who have sleep difficulties.10] We tried to intervene through several methods like cognitive behavioral methods- like reading bedtime stories and took several actions like reducing screen time and promoting physical exercises- like playing on the field. Apart from that, we asked the parents to take several steps like singing lullabies, giving gentle caress when the children seem to show aberrant behaviors. The result looks really good after the intervention. The treatment helped reduce sleep disruption for all individuals, and the benefits were largely sustained at the follow-up. Children with sleep problems (both those with and without ASD) show more challenging behaviors than children without sleep problems Children with sleep problems (both those with and without ASD) show more challenging behaviors than children without sleep problems. Several researches on behavioral intervention on children with ASD having sleep disturbance have been made by many scholars. In Bangladesh, people with ASD are seldom treated with care and respect. In our study, we have taken complete care of our participants. Out of the 41 children, 27 were male and 14 were female. We recorded their percentage and tried to observe the two data types separately. A similar take on different research on sleep disturbances have been made by Elizabeth J. Halstead and her fellow researchers, where they took 100 participants and divided them into separate age groups for better observation.11] This research was also done through total parental observation and care. Another research done by Sarah A. Schoen observed the changes in sleep disturbances from Pretest to Intervention. [12] Our study does the same, by observing and jotting down the comparison between the previous sleep experience with the present one with intervention. Children with sleep problems show more challenging behaviors than children without sleep problems.[13] Sleep hygiene methods, which focus on altering the environment to support a normal sleep-wake cycle, are effective treatments in improving sleep onset and maintenance in ASD. The fundamental concepts of sleep hygiene include choosing a suitable bedtime and routine, limiting television viewing, and limiting emotional and behavioral stimuli at night. Given the links between insufficient sleep, increased daytime problem behaviors, and 
Annals of International Medical and Dental Research

E-ISSN: 2395-2822 | P-ISSN: 2395-2814

Vol-8, Issue-1 | January-February 2022

DOI: 10.53339/aimdr.2022.8.1.41

Page no- 325-332 | Section- Research Article (Paediatric Neurosciences)

parental stress in ASD, there is a significant need to create effective sleep therapies that are tailored to a child's cognitive and developmental level.

\section{Limitations of the study}

Because of resource and time limits, this study followed convenient purposive sampling technique with smaller sample size than expected. So, study may not reflect the total scenario of whole Bangladesh. This is only a sample size of the entire children with ASD population in Bangladesh. This doesn't necessarily define the entirety of the population of children with ASD in Bangladesh.

\section{CONCLUSIONS}

The study has tried and succeeded to an extent to intervene in the sleep dysfunction process of children with ASD in a tertiary care hospital. However, there is still much to learn about the

\section{REFERENCES}

1. Hodges H, Fealko C, Soares N. Autism spectrum disorder: definition, epidemiology, causes, and clinical evaluation. Transl Pediatr. 2020;9(Suppl 1):S55-S65. doi:10.21037/tp.2019.09.09

2. Cortesi F, Giannotti F, Ivanenko A, Johnson K. Sleep in children with autistic spectrum disorder. Sleep Med. 2010;11(7):659-64. doi: 10.1016/j.sleep.2010.01.010.

3. Souders MC, Zavodny S, Eriksen W, Sinko R, Connell J, Kerns C, Schaaf R, Pinto-Martin J. Sleep in Children with Autism Spectrum Disorder. Curr Psychiatry Rep. 2017;19(6):34. doi: 10.1007/s11920017-0782-x.

4. Papadopoulos N, Sciberras E, Hiscock H, Mulraney M, McGillivray J, Rinehart N. The Efficacy of a Brief Behavioral Sleep Intervention in School-Aged Children With ADHD and Comorbid Autism clinical efficacy of these types of behavioral interventions in children with ASD who have sleep disruption.

Although there is evidence of sleep issues getting solved or lessened with intervention in children with ASD, these findings require additional research. Gaining more particular insight into the individual character of sleep issues in ASD opens up a new route for creating treatments, as sleep is an area with the possibility for remediation. However, research too far has failed to offer clear data concerning the link between sleep and behaviors found in children with ASD. This study emphasizes the need of developing sleep profiles for children with ASD and relating various components of their symptom profile to their sleep impairments (and vice versa). As a result of this knowledge, researchers will be able to conduct studies that will, ideally, enhance long-term outcomes in the future.

Spectrum Disorder. J Atten Disord. 2019;23(4):341350. doi: $10.1177 / 1087054714568565$.

5. Halstead EJ, Joyce A, Sullivan E, et al. Sleep Disturbances and Patterns in Children With Neurodevelopmental Conditions. Front Pediatr. 2021;9:637770. doi:10.3389/fped.2021.637770

6. Vriend JL, Corkum PV, Moon EC, Smith IM. Behavioral interventions for sleep problems in children with autism spectrum disorders: current findings and future directions. J Pediatr Psychol. 2011;36(9):1017-29. doi: 10.1093/jpepsy/jsr044.

7. Hollway JA, Aman MG. Sleep correlates of pervasive developmental disorders: a review of the literature. Res Dev Disabil. 2011;32(5):1399-421. doi: 10.1016/j.ridd.2011.04.001.

8. Liu X, Hubbard JA, Fabes RA, Adam JB. Sleep disturbances and correlates of children with autism spectrum disorders. Child Psychiatry Hum Dev. 2006;37(2):179-91. doi: 10.1007/s10578-006-0028-3. 
Annals of International Medical and Dental Research

E-ISSN: 2395-2822 | P-ISSN: 2395-2814

Vol-8, Issue-1 | January-February 2022

DOI: $10.53339 /$ aimdr.2022.8.1.41

Page no- 325-332 | Section- Research Article (Paediatric Neurosciences)

9. Cohen S, Conduit R, Lockley SW, Rajaratnam SM, Cornish KM. The relationship between sleep and behavior in autism spectrum disorder (ASD): a review. J Neurodev Disord. 2014;6(1):44. doi: 10.1186/1866-1955-6-44.

10. Vohra R, Madhavan S, Sambamoorthi U, St Peter C. Access to services, quality of care, and family impact for children with autism, other developmental disabilities, and other mental health conditions. Autism. 2014;18(7):815-826. doi:10.1177/1362361313512902

11. McLay L, France K, Blampied N, Hunter J. Using functional behavioral assessment to treat sleep problems in two children with autism and vocal stereotypy. Int J Dev Disabil. 2017;65(3):175-184. doi:10.1080/20473869.2017.1376411

12. Schoen S, Man S, Spiro C. A Sleep Intervention for Children with Autism Spectrum Disorder: A Pilot Study. J Occup Ther. 2017;5(2):1-11.

13. Allik H, Larsson JO, Smedje H. Sleep patterns of school-age children with Asperger syndrome or high-functioning autism. J Autism Dev Disord. 2006;36(5):585-95. doi: 10.1007/s10803-006-0099-9.

Source of Support: Nil, Conflict of Interest: None declared 